\title{
Dissociable Roles of Mid-Dorsolateral Prefrontal and Anterior Inferotemporal Cortex in Visual Working Memory
}

\author{
Michael Petrides \\ Montreal Neurological Institute and Department of Psychology, McGill University, Montreal, Quebec H3A 2B4, Canada
}

\begin{abstract}
Functional neuroimaging in human subjects and studies of monkeys with lesions limited to the mid-dorsolateral (MDL) prefrontal cortex have shown that this specific region of the prefrontal cortex is involved in visual working memory, although its precise role remains a matter of debate. The present study compared the effect on visual working memory of lesions restricted to the mid-dorsolateral prefrontal cortex of the monkey with that of lesions to the anterior inferotemporal cortex, a region of the temporal cortex specialized for visual memory. Increasing the delay during which information had to be maintained in visual working memory impaired performance after lesions of the anterior inferotemporal cortex, but not after mid-dorsolateral prefrontal lesions. By contrast, increasing the number of stimuli that had to be monitored impaired the performance of animals with mid-dorsolateral prefrontal lesions, but not that of animals with
\end{abstract}

There is considerable evidence from functional neuroimaging in human subjects (for review, see Owen, 1997; D'Esposito et al., 1998; Petrides, 2000) and studies of monkeys with lesions limited to the mid-dorsolateral (MDL) prefrontal cortex (Petrides, 1991, 1995 ) that this region is involved in visual working memory. But what precisely is the nature of the involvement of the middorsolateral prefrontal cortex in visual working memory? Does this cortical region play a primary role in the maintenance of information in working memory or is it involved in the executive process of keeping track (i.e., monitoring) of multiple events in working memory? It has been argued (Petrides, 1991, 1994) that the role of the mid-dorsolateral prefrontal cortex lies in the executive process of monitoring information in working memory.

The present investigation tested in the strictest possible manner the above two interpretations of the nature of the impairment in visual working memory after mid-dorsolateral prefrontal lesions. This was achieved by manipulating the difficulty of the same visual working memory task in two independent ways: (1) by increasing the delay, i.e., the time during which information must be maintained while keeping the number of stimuli to be monitored low and constant, and (2) by increasing the number of stimuli that must be monitored while keeping the delay low and constant. In addition, the performance of the animals with mid-dorsolateral prefrontal lesions was compared with that of animals with anterior inferotemporal lesions. This lateral temporal region is known to be the last stage in the specialized occipitotemporal system for the processing of visual pattern (object) information and has been shown to be critical for visual object memory (Mishkin, 1982). Thus, not only was the effect of lesions to the mid-dorsolateral prefrontal cortex tested under conditions that independently manipulated delay and number of stimuli to be monitored, but it was simultaneously

\footnotetext{
Received June 2, 2000; revised July 25, 2000; accepted July 25, 2000.

This study was supported by the Natural Sciences and Engineering Council of Canada (Grant A7466). I thank K. Morris for testing the animals.

Correspondence should be addressed to Dr. Michael Petrides, Montreal Neurological Institute, 3801 University Street, Montreal, Quebec, H3A 2B4 Canada. E-mail: petrides@ego.psych.mcgill.ca.

Copyright (C) 2000 Society for Neuroscience $0270-6474 / 00 / 207496-08 \$ 15.00 / 0$
}

anterior inferotemporal lesions. This demonstration of a double dissociation between the effects of these two lesions provides strong evidence that the role of the mid-dorsolateral prefrontal cortex in visual working memory does not lie in the maintenance of information per se, but rather in the executive process of monitoring this information. In addition, the present study demonstrated that lesions limited to area 9, which constitutes the superior part of the mid-dorsolateral prefrontal region, give rise to a mild impairment in the monitoring of information, whereas lesions of the complete mid-dorsolateral prefrontal region yield a very severe impairment.

Key words: dorsolateral prefrontal cortex; inferotemporal cortex; visual working memory; monkey; lesions; monitoring; control process

compared with that of lesions to the part of the cortex that is considered to be specialized for visual object memory.

The mid-dorsolateral prefrontal region can be divided into a lower granular part in which layer IV is well developed (areas 46D and 9/46D) and a superior dysgranular part that consists of a related type of cortex but in which the granular layer IV is not as well developed (area 9) (see Fig. 1). The granular part occupies the lower half of the mid-dorsolateral prefrontal region up to and including the lip of the sulcus principalis [for details, see Petrides and Pandya $(1994,1999)]$. In the present investigation, one group of monkeys had lesions that included both the granular and dysgranular parts of the mid-dorsolateral prefrontal region, and another group had lesions confined to the superior dysgranular part of this region.

The present experiment was reported at the 1998 Meeting of the Society for Neuroscience (Petrides, 1998).

\section{MATERIALS AND METHODS}

\section{Subjects and surgical procedure}

The subjects were 16 adult male monkeys (Macaca nemestrina) weighing $4.5-9.5 \mathrm{~kg}$ at the time of surgery. All operations were performed by standard aseptic operating techniques for the subpial aspiration of cortical tissue. The animals were first anesthetized with ketamine $(15 \mathrm{mg} / \mathrm{kg}, \mathrm{i} . \mathrm{m}$.) and then given the minimal dose of intravenous barbiturate (Nembutal) necessary to induce a deep state of anesthesia. Supplementary doses were administered during the operation as needed. Antibiotics were administered postoperatively to prevent infection. All operations were bilateral and were performed in one-stage.

There were two groups of animals with mid-dorsolateral prefrontal lesions. Three animals had complete lesions of the mid-dorsolateral region and constituted the MDL group. These lesions were intended to involve both the lower granular part (areas 46D and 9/46D) and the superior dysgranular part (area 9) of the mid-dorsolateral region (Figs. 1B, 2). Three animals had lesions that were confined to the superior dysgranular part of the mid-dorsolateral (SMD) prefrontal region, i.e., area 9, and these animals constituted the SMD group (Figs. $1 C, 3$ ). Four animals served as the normal control (NC) group. In addition, three animals had lesions confined to the posterior dorsolateral prefrontal region around the arcuate sulcus and formed the periarcuate (PA) group (Fig. 4). These periarcuate lesions, which had previously been shown not to impair visual working memory (Petrides, 1991, 1995), although they yield severe impairments in conditional learning (Petrides, 1987), served as an additional operated control group. Three monkeys [anteroventral inferotemporal (AIT) group] 


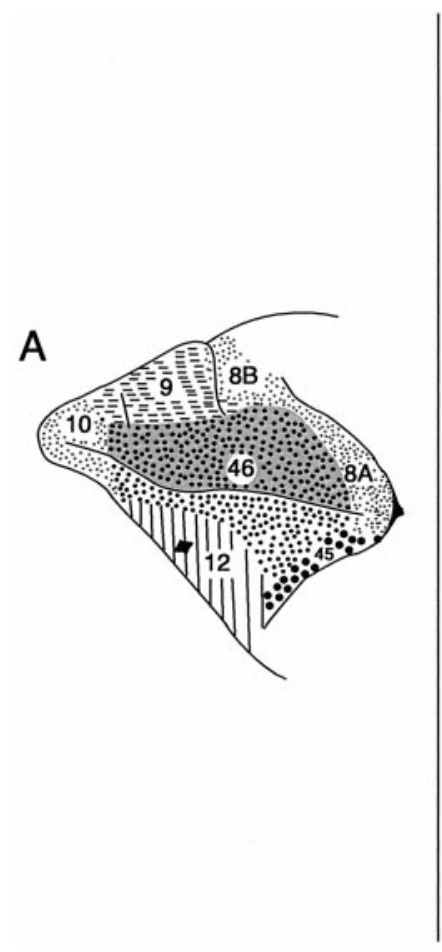

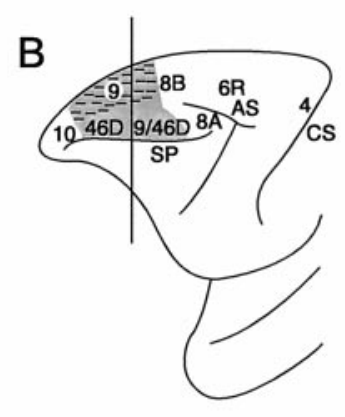
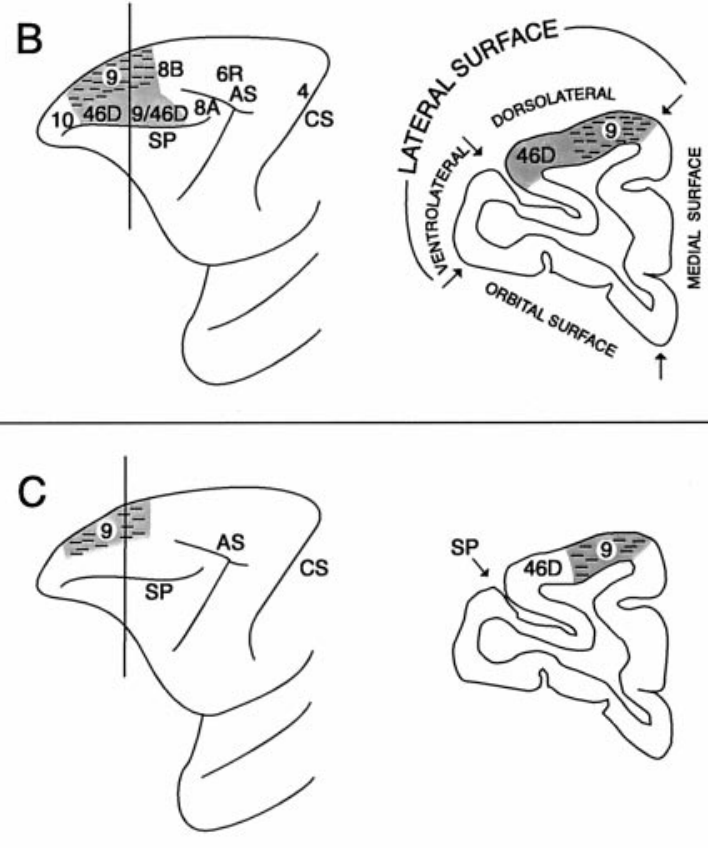

Figure 1. A, The classic cytoarchitectonic map of Walker (1940). Note that the dorsal part of area 46 (dark gray dotted area), which constitutes the granular part of the mid-dorsolateral prefrontal cortex, extends for a considerable distance above the sulcus principalis. Area 9, which occupies the superior part of the middorsolateral prefrontal region, exhibits a less developed granular layer IV. Walker (1940) pointed out that the region he labeled as "area 46" is not homogeneous, but he did not subdivide it further. For instance, dorsal area 46 that lies within the mid-dorsolateral prefrontal region above the sulcus principalis (dark gray dotted area) is architectonically distinct from the sulcal part of area 46 that lines the inner part of the sulcus principalis [for details, see Petrides and Pandya (1994, 1999)]. B, Illustration of the intended complete mid-dorsolateral lesion (i.e., the MDL lesion). This lesion was to include both the granular (dorsal areas 46 and 9/46) and dysgranular (area 9) parts of the mid-dorsolateral region. In a comparative architectonic study of the macaque and the human prefrontal cortex, we noted that Walker's area 9 is comparable with area 9 on the superior frontal gyrus of the human brain, whereas the dorsal part of area 46 of Walker is comparable with dorsal areas 46 and 9/46 of the human brain that lie on the middle frontal gyri [for details, see Petrides and Pandya

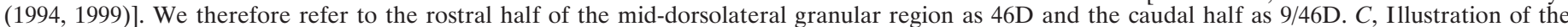

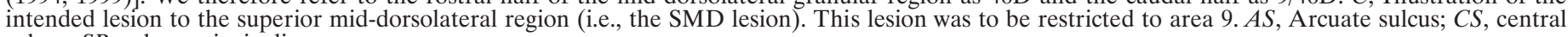
sulcus; $S P$, sulcus principalis.
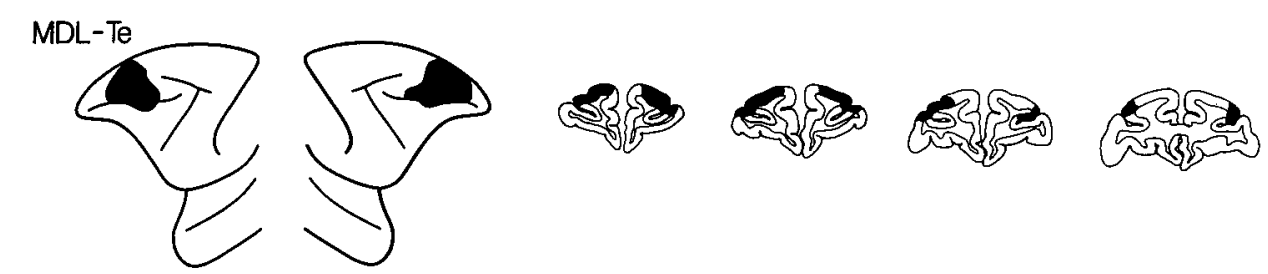

MDL-Mi
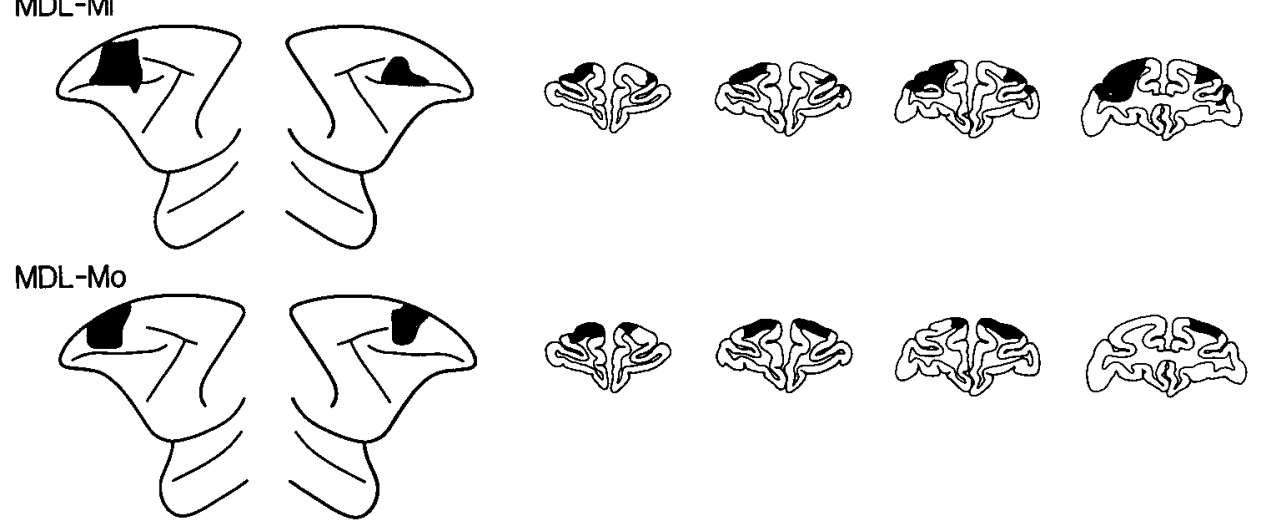

Figure 2. Extent of the lesions of the middorsal part of the lateral frontal cortex (MDL lesions) depicted (black) on standard diagrams of the lateral surface of the anterior part of the brain of the monkey and on standard drawings of coronal sections. Left hemisphere is shown on the left side, both for the lateral surface diagrams and for the coronal sections. The approximate stereotaxic levels for the coronal sections shown are, from left to right, $+36,+34,+32,+30$. $M D L-T e, M D L-M i$, and $M D L-M o$ refer to individual monkeys. had lesions confined to the anterior inferotemporal cortex, which has been shown to play a role in visual memory (Figs. 5, 6).

The MDL, PA, and NC groups were the same animals that had been studied previously (Petrides, 1991, 1995). The present experiment was administered at the end of Experiment 9 in Petrides (1995), $\sim 3$ years after operation. The animals forming the SMD and AIT groups were additional subjects and had a testing history similar to that of the other groups.

$M D L$ group. This group comprised three monkeys that were given bilateral ablation of the mid-dorsal part of the lateral frontal cortex (Figs. $1 B, 2$ ). These lesions were restricted within the granular (areas 46D and 9/46D) and dysgranular (area 9) part of the mid-dorsolateral frontal region. They are referred to here as the MDL frontal lesions because they were intended to extend from above the middle sector of the sulcus principalis to the midline. These lesions were intended to spare the most rostral part of the dorsolateral frontal cortex, which is part of the frontopolar cortex (i.e., area 10), and, posteriorly, the cortex surrounding the upper branch of the arcuate sulcus, as far as the midline (i.e., areas $8 \mathrm{~A}, 8 \mathrm{~B}$, and rostral 6 ), as well as the cortex surrounding the posteriormost part of the sulcus principalis (in the concavity of the arcuate sulcus) that is part of area 8A, in terms of both architecture and connections (Pandya and Kuypers, 1969; Pandya et al., 1971; Barbas and Mesulam, 1981; Petrides and Pandya, 1994, 1999). In making these lesions, great care was also taken not to damage or undercut the connections of the inferior frontal convexity, i.e., the ventrolateral frontal cortex that lies below the sulcus principalis.

SMD group. Three monkeys were given bilateral ablation of the superior half of the mid-dorsolateral frontal cortex, i.e., area 9 (Figs. 1C, 3). These area 9 lesions were intended to spare the cortex of the sulcus principalis and the granular area just above it (i.e., areas 46D and 9/46D), the most rostral part of the dorsolateral frontal cortex, which is part of the frontopolar cortex (i.e., area 10), and posteriorly, the cortex surrounding the upper branch of the arcuate sulcus, as far as the midline, (i.e., areas $8 \mathrm{~A}, 8 \mathrm{~B}$, and rostral 6).

$P A$ group. Three animals were given a bilateral ablation of the PA region, i.e., the posterior part of the dorsolateral frontal cortex (Fig. 4). These 
SMD-1

Figure 3. Extent of the lesions confined to the superior part of the mid-dorsolateral prefrontal region (SMD lesions) depicted (black) on standard diagrams of the lateral surface of the anterior part of the brain of the monkey and on standard drawings of coronal sections. Left hemisphere is shown on the left side, both for the lateral surface diagrams and for the coronal sections. The approximate stereotaxic levels for the coronal sections shown are, from left to right, $+36,+34,+32,+30$. SMD-1, SMD-2, and $S M D-3$ refer to individual monkeys.

SMD-2
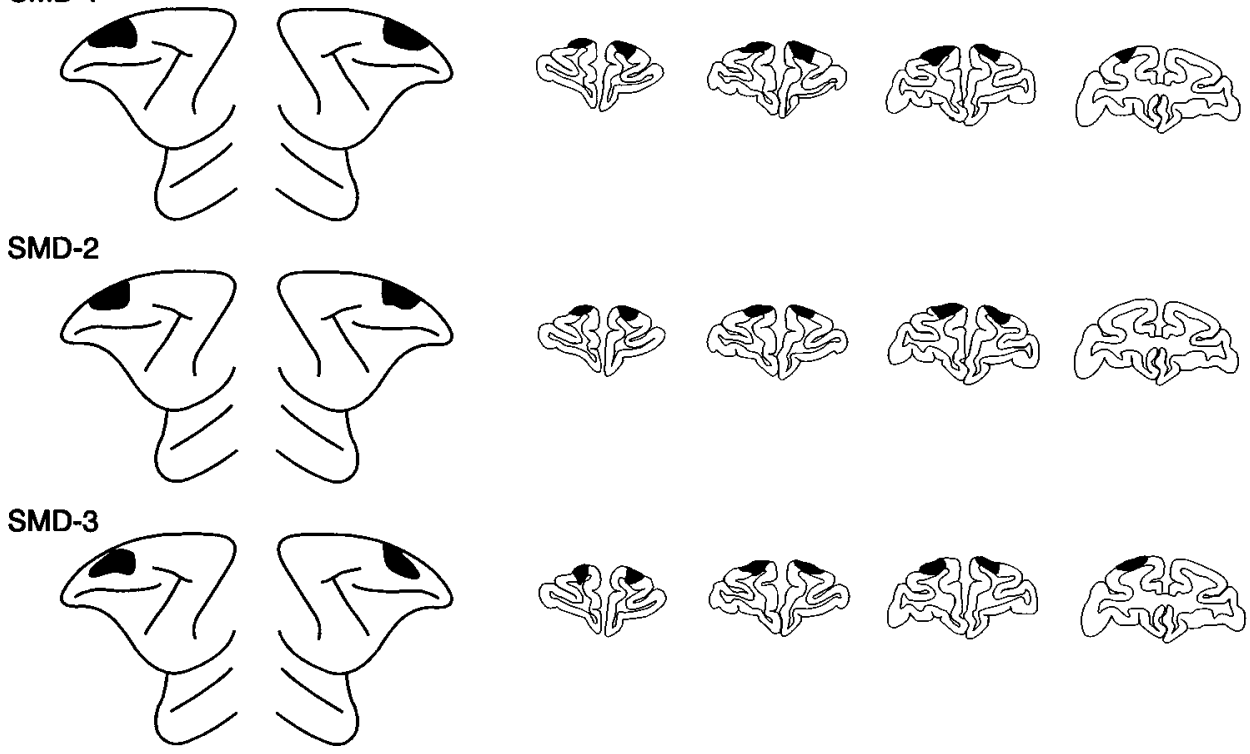

lesions were to include the cortex surrounding and lying within the superior ramus, the spur, and the uppermost part of the inferior ramus of the arcuate sulcus. In other words, rostral area 6 , which lies within the posterior bank of the arcuate sulcus, and area 8, within the anterior bank of the arcuate sulcus, were to be removed. In making these lesions, great care was taken not to damage or undercut the more rostrally located frontal cortex.

AIT group. Three monkeys had lesions confined to the anterior part of the inferotemporal cortex, extending from the rostralmost part of the superior temporal sulcus to the tip of the occipitotemporal sulcus (Figs. 5, 6). The lesion was intended to ablate areas TE1 and TE2, as defined by Pandya (Pandya and Yeterian, 1985). To gain access to the ventrolateral surface of the temporal lobe, the zygomatic arch was removed, and the muscle temporalis was reflected, permitting the opening of a large bone flap that extended to the base of the temporal fossa.

NC group. The four monkeys that comprised this group were not subjected to any operation.

\section{Histological procedure}

At the completion of the present and other experiments, the operated animals were killed with an overdose of Nembutal, and their brains were removed and fixed in a $10 \%$ formalin solution. A macroscopic examination of the brains, which included drawings of the lesions as seen from the surface of the brain, was carried out. Frozen sections of the part of each brain that included the lesion were then cut at $60 \mu \mathrm{m}$, and every sixth section was kept for staining with thionin. A microscopic examination of the stained sections was conducted, and drawings of the lesions were made (Figs. 2-6).

\section{Testing}

All testing was performed in a Wisconsin General Testing Apparatus, which consists of a compartment where the monkey is held and a testing area. An opaque screen can be interposed between these two compartments to occlude the monkey's view of the testing area. The animals were tested for $5 \mathrm{~d}$ during each week and were rewarded with banana-flavored food pellets for correct responses. All animals were highly trained on nonspatial visual self-ordered and externally ordered working memory tasks. The precise testing history before the present experiment of the animals comprising the MDL, PA, and NC groups can be found in Petrides $(1991,1995)$. The animals comprising the SMD and AIT groups had also received extensive testing on the same nonspatial visual self-ordered tasks administered to the other groups.

In the nonspatial visual self-ordered tasks, the animal is faced, during successive trials, with the same set of stimuli (e.g., objects A, B, and C) and has to select a different one of these stimuli on each trial until all stimuli have been selected once (Petrides, 1995). The relative position of the stimuli changes from trial to trial to ensure that the animal is guided by memory of the objects and not the locations that they occupy. In other words, correct performance (i.e., the response leading to reward) is not to select a stimulus that had been chosen on an earlier trial because reselection of that stimulus would not lead to reward. Thus, training on the self-ordered tasks teaches the monkey that the same set of stimuli will reappear on all trials and that these stimuli must be selected only once during the successive trials. Thus, successful performance depends on attending to all of the stimuli in the set and carefully monitoring successive
Figure 4. Extent of the periarcuate $(P A)$ lesions, i.e., the lesions of the posterior dorsolateral frontal cortex, depicted (black) on standard diagrams of the lateral surface of the anterior part of the brain of the monkey and on standard drawings of coronal sections. Left hemisphere is shown on the left side, both for the lateral surface diagrams and for the coronal sections. The approximate stereotaxic levels for the coronal sections shown are, from left to right, $+26,+24,+22,+20$. $P A-C h, P A-$ $S p$, and $P A-S y$ refer to individual monkeys.
$\mathrm{PA}-\mathrm{Ch}$
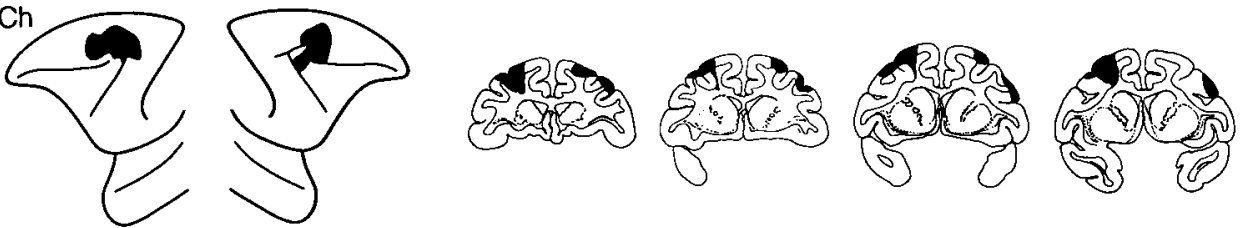

$\mathrm{PA}-\mathrm{Sp}$
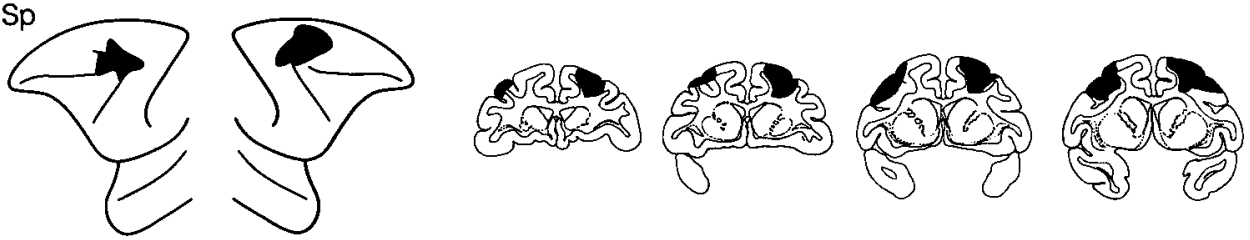

PA-Sy
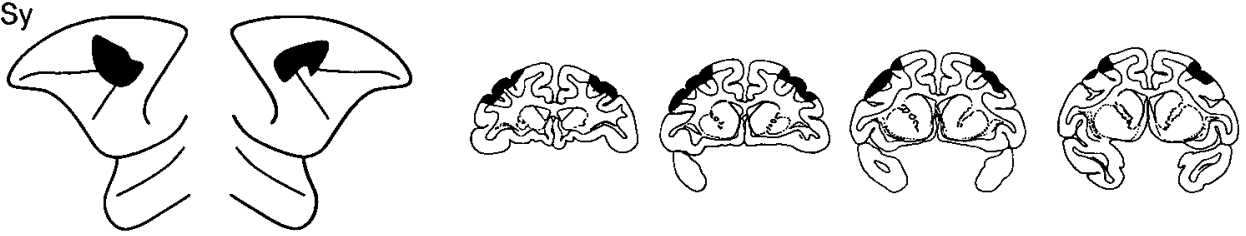


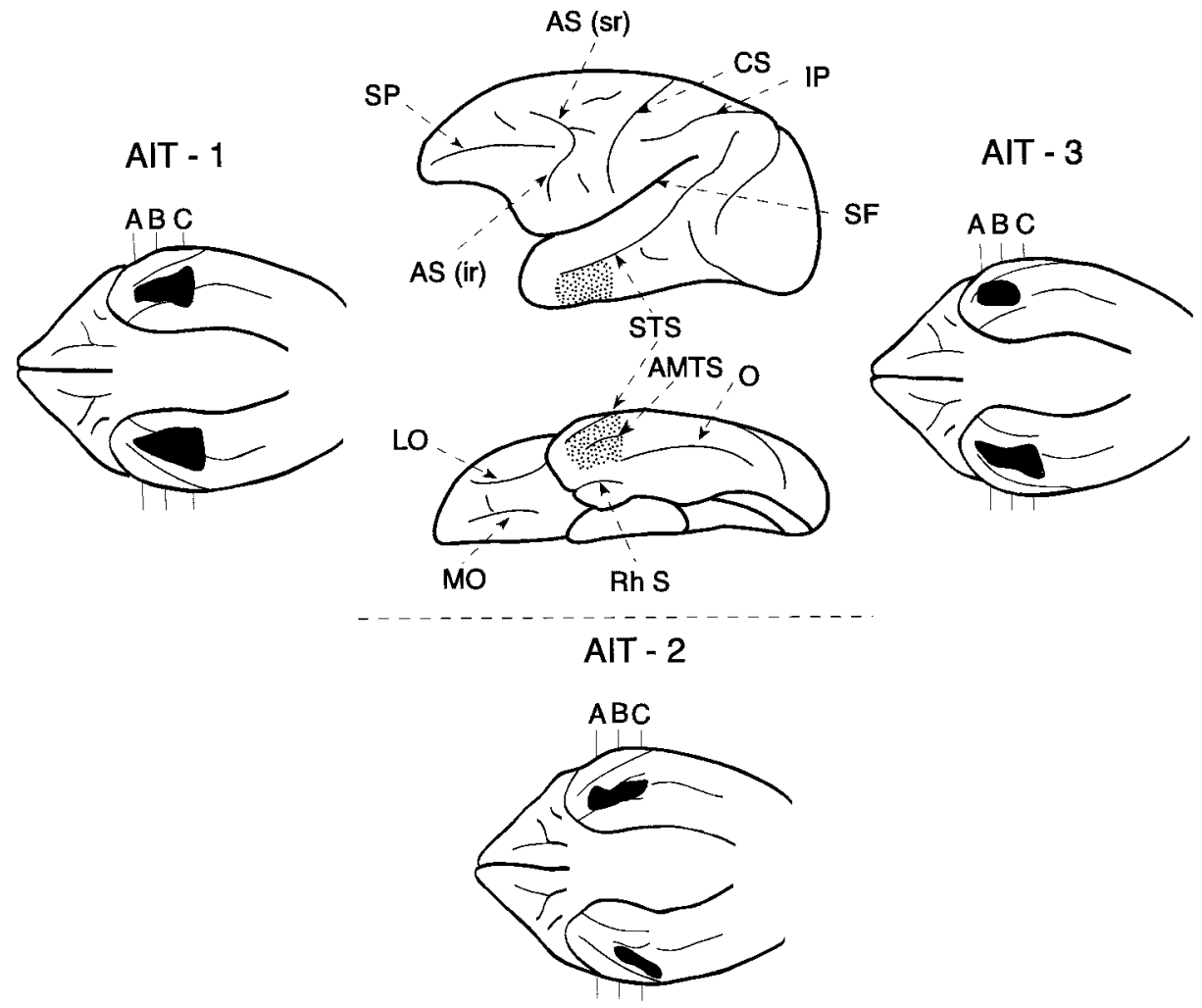

Figure 5. Schematic diagram of the lateral and inferior surfaces of the left hemisphere of the monkey to illustrate the location (area indicated by the dots) of the anteroventral inferotemporal region (areas TE1 and TE2). This region extends from the tip of the superior temporal sulcus (STS) to the tip of the occipitotemporal sulcus $(O)$, excluding the perirhinal cortex along the rhinal sulcus $(R h S)$. Note that the rostral part of the STS can be viewed on both the lateral and the inferior surfaces of the brain. The lesions of the three monkeys (AIT-1, $A I T-2$, and $A I T-3$ ) that constituted this group are shown (black) on the diagrams of the inferior surface of the brain because only on this view can the entire extent of this region be visualized. $A, B$, and $C$ indicate the level of the coronal sections shown in Figure 6. AMTS, Anterior middle temporal sulcus; $A S$ (ir), inferior ramus of the arcuate sulcus; $A S(s r)$, superior ramus of the arcuate sulcus; $C S$, central sulcus; $I P$, intraparietal sulcus; $L O$, lateral orbital sulcus; $M O$, medial orbital sulcus; $R h S$, rhinal sulcus; $S F$, Sylvian fissure; $S P$, sulcus principalis; $S T S$, superior temporal sulcus; $O$, occipitotemporal sulcus.

choices (i.e., noting which stimuli from the recurring set have been selected and which have yet to be selected). This principle was essential for the experiment that is described below.

For the present experiment, the standard version of the self-ordered task (Petrides, 1995) was modified so that the number of stimuli to be monitored and the delay during which information must be maintained could be manipulated independently. Testing consisted of a series of pairs of presentation and test trials. The presentation trial was identical to the first trial on the standard self-ordered task; namely, the animal was faced with a familiar set of objects (Fig. 7). The stimuli were placed over small white plaques that covered food wells on the test board, and under each plaque there was a reward. The animal chose one of the objects from the array by displacing a white plaque to obtain the reward that lay under it. In the standard version of the self-ordered task, the same stimuli reappear on successive trials until all are selected once. In the present modified version, only one test trial followed the presentation trial. On this test trial (Fig. 7), the animal was faced with the stimulus it had chosen on the preceding presentation trial and one of the stimuli that it had not chosen. As in all previous training of these animals, the correct (i.e., rewarded) response on this test trial was to select the stimulus not previously chosen.

In one set of conditions, the length of the delay between the presentation trials and the test trials was varied (i.e., 10, 90, and $120 \mathrm{sec}$ ), but the stimulus set during the presentation trials was always two objects. In the other set of conditions, the size of the stimulus set was varied during the presentation trials (i.e., two, three, four, or five objects), but the delay between the presentation trials and the test trials was held constant at 10 sec. The order of administration of the two parts of the experiment (set stimulus size manipulation vs delay manipulation) was counterbalanced within each group. Ten pairs of presentation and test trials were administered per day. The presentation trial was initiated by the raising of an opaque screen to display to the animal a set of small objects. In the presentation trials of the conditions in which the size of the stimulus set was varied, two, three, four, or five objects (depending on the particular condition being administered) were placed in a row over small white plaques covering food wells on the test board (Fig. 7). Each one of these objects covered a reward. The animal was allowed to displace any one of these objects to obtain the reward that was hidden under it, and the opaque screen was then lowered. After a delay period of $10 \mathrm{sec}$, the opaque screen was again raised to initiate the test trial. On the test trial, in all conditions, the monkey was faced with only two stimuli, which were placed over the white plaques covering two food wells of the testing board. One of these stimuli was the object that the animal had chosen on the preceding presentation trial, and the other was taken randomly from the stimuli that the animal had not selected on the presentation trial. For instance, if three objects (e.g., A, B, C) were shown on the presentation trial and the animal had chosen object $A$, then on the test trial the animal would be faced with a choice between A and one of the other objects (e.g., C). Only the stimulus not selected on the preceding presentation trial covered a reward on the test trial ( $\mathrm{C}$ in the example above), and the monkey was therefore rewarded only if it selected that stimulus. Displacing the plaque with the stimulus that had been selected on the presentation trial did not result in reward and was scored as an error. The left/right position of the two stimuli on the test trials was determined randomly but with the restriction that over the 10 daily test trials the baited object would occur an equal number of times over the left and right positions and that no position would be baited for more than three consecutive test trials. The animals were tested for a total of 200 pairs of presentation and test trials per stimulus-set condition (i.e., a total of 800 pairs of trials).

In the part of the experiment in which the length of delay was manipulated, the testing procedure was identical to that described above, except that only two objects were presented on the presentation trial, and on the test trial the animal was faced with a choice between the object it had chosen and the one that it had not chosen on the preceding presentation trial. The conditions in this part of the experiment differed only in terms of the length of the delay; i.e., the delay between the presentation and test trials could be 10,90 , or $120 \mathrm{sec}$. The animals were tested for a total of 200 pairs of presentation and test trials per delay condition (i.e., a total of 600 pairs of trials).

\section{RESULTS}

The effect of increasing the delay (from 10 to $90 \mathrm{sec}$ and then to 120 sec) while keeping the number of stimuli to be monitored low (i.e., two stimuli) can be seen in Figure 8 . The scores of individual monkeys in the three delay conditions can be found in Table 1. Inspection of the performance of normal animals (NC group) shows that increasing the delay was effective in making the task more difficult for the normal animals ( $p<0.001$ for $10 \mathrm{sec}$ or $90 \mathrm{sec}$ vs $120 \mathrm{sec})$. A two-way ANOVA with repeated measures revealed a significant Group $\times$ Delay Interaction $\left(F_{(8,22)}=6.26, p<0.001\right)$. Further exploration of this effect with the Newman-Keuls test revealed that in comparison with the control animals, the group with anterior inferotemporal lesions was significantly impaired at the $90 \mathrm{sec}(p<0.001)$ and $120 \mathrm{sec}(p<0.001)$ delays; none of the other groups differed from the control group. Thus, the animals with the mid-dorsolateral prefrontal lesions (MDL and SMD groups) performed as well as the normal control animals and those with the periarcuate lesions. Delay affected differentially only the performance of the animals with lesions to the anterior inferotemporal cortex; these animals performed at chance on the $120 \mathrm{sec}$ delay condition.

The effect of increasing the number of stimuli that the animals had to monitor while keeping the delay short (i.e., $10 \mathrm{sec}$ ) is shown 

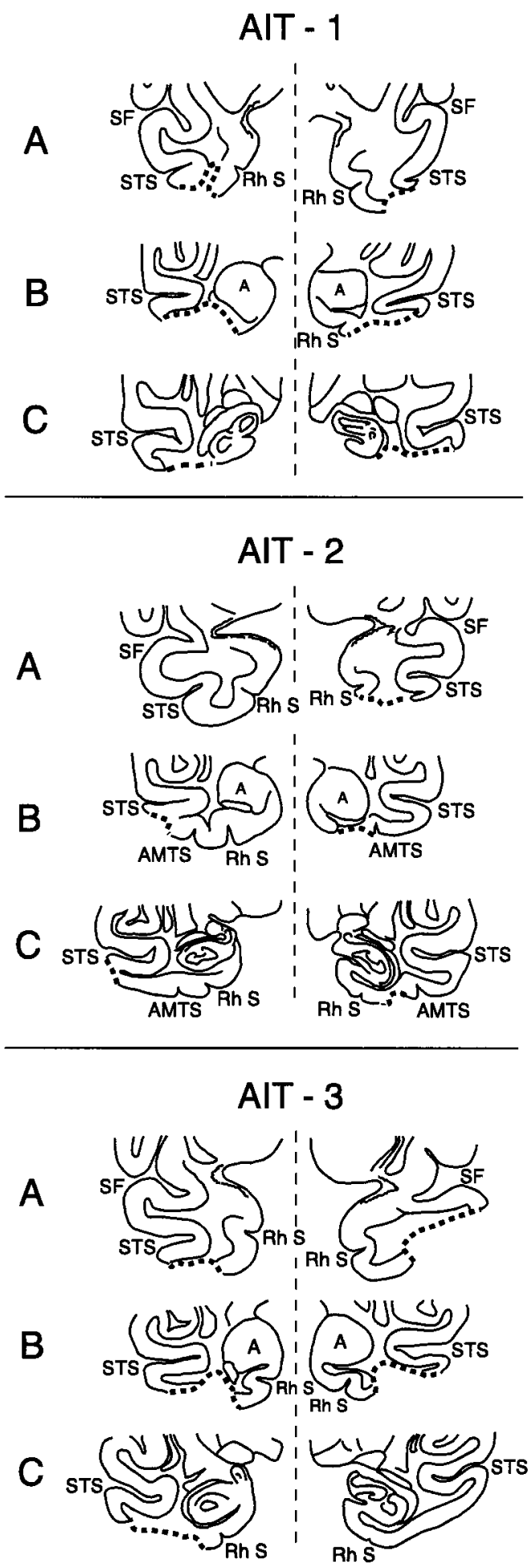

Figure 6. Coronal sections through the anterior temporal lobe to illustrate the lesions (depicted by the thick dotted line) in the anteroventral part of the inferotemporal region in the three monkeys $(A I T-1, A I T-2$, and $A I T-3)$ that had lesions to this region. The levels of sections $A, B$, and $C$ are indicated in Figure 5 on the reconstructions of the inferior surfaces of the brains of the three monkeys. Left hemisphere is shown on the right side for the coronal sections. $A$, Amygdala; $A M T S$, anterior middle temporal sulcus; $R h$ $S$, rhinal sulcus; $S F$, Sylvian fissure; $S T S$, superior temporal sulcus.

in Figure 9. The scores of individual monkeys can be found in Table 2. As the size of stimulus set from which the two test stimuli were drawn increased, the performance of the two groups with mid-dorsolateral prefrontal lesions deteriorated, whereas that of the animals with anterior inferotemporal lesions and those with
2 STIMULUS SET

A

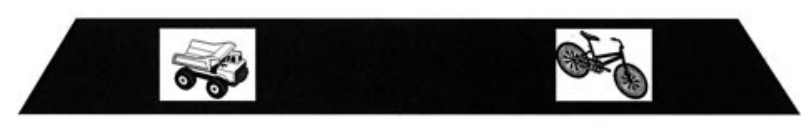

B

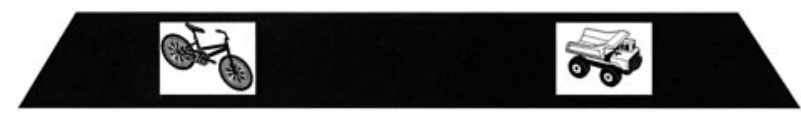

\section{STIMULUS SET}

A

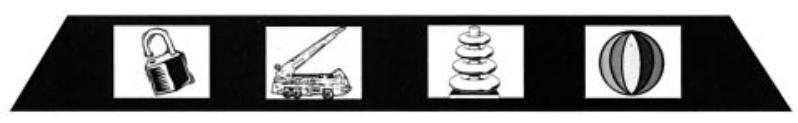

B

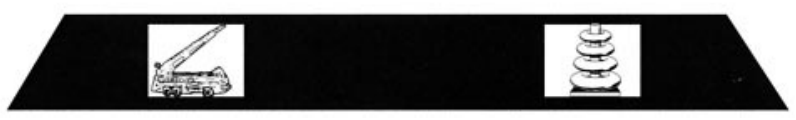

Figure 7. Schematic diagram of the experimental arrangement in the condition in which stimulus set size was manipulated. The sets with two and four stimuli are illustrated. Note that on the presentation trials $(A)$, all of the stimuli comprising the particular set are displayed in front of the monkey. Once the animal has selected one of these objects, a test trial $(B)$ is administered $10 \mathrm{sec}$ later, during which the object selected is paired with one of the objects not selected.

\section{Group x Delay Interaction}

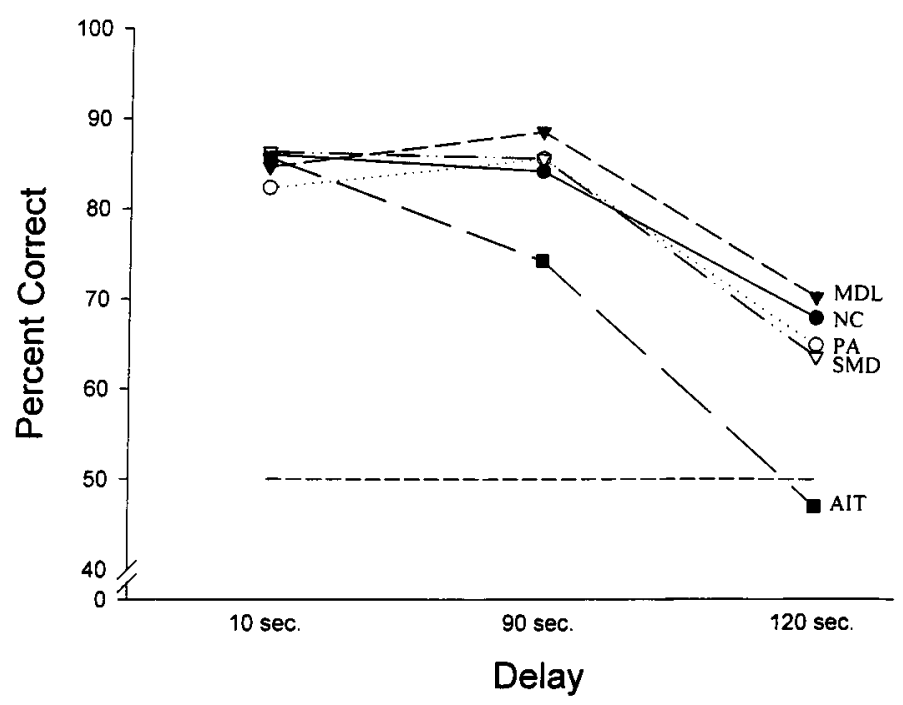

Figure 8. Graph showing the mean performance of each group across the three delays. The level of chance performance $(50 \%)$ is indicated by the horizontal dotted line. AIT, Group with lesions to the anteroventral inferotemporal region; $M D L$, group with damage to both the lower and upper parts of the mid-dorsolateral prefrontal cortex; $N C$, normal control group; $P A$, group with periarcuate lesions; $S M D$, group with damage confined to the superior part of the mid-dorsolateral prefrontal cortex (area 9).

posterior dorsolateral prefrontal lesions (i.e., the periarcuate region) was not significantly different from the normal control performance $\left(\right.$ Group $\times$ Stimulus Set Interaction: $F_{(12,33)}=6.48, p<$ $0.001)$. The significance levels of the interactions reported above were also obtained using the Greenhouse-Geisser conservative degrees of freedom. The animals with the lesions of the complete mid-dorsolateral prefrontal region (MDL group) were more impaired than the animals with the lesions restricted to its upper part 
Table 1. Percentage correct performance on the delay conditions

\begin{tabular}{|c|c|c|c|}
\hline Group & $10 \mathrm{sec}$ & $90 \mathrm{sec}$ & $120 \mathrm{sec}$ \\
\hline \multicolumn{4}{|l|}{$\mathrm{NC}$ group } \\
\hline NC1 & 84 & 87 & 70 \\
\hline $\mathrm{NC} 2$ & 88 & 82 & 65 \\
\hline $\mathrm{NC} 3$ & 82 & 84 & 68 \\
\hline $\mathrm{NC} 4$ & 90 & 84 & 69 \\
\hline \multicolumn{4}{|l|}{ PA group } \\
\hline PA1 & 80 & 88 & 66 \\
\hline PA2 & 84 & 84 & 69 \\
\hline PA3 & 83 & 85 & 60 \\
\hline \multicolumn{4}{|c|}{ MDL group } \\
\hline MDL1 & 90 & 87 & 70 \\
\hline MDL2 & 84 & 88 & 69 \\
\hline MDL3 & 80 & 91 & 72 \\
\hline \multicolumn{4}{|c|}{ SMD group } \\
\hline SMD1 & 88 & 84 & 64 \\
\hline SMD2 & 90 & 84 & 67 \\
\hline SMD3 & 81 & 89 & 60 \\
\hline \multicolumn{4}{|c|}{ AIT group } \\
\hline AIT1 & 84 & 73 & 44 \\
\hline AIT2 & 91 & 76 & 46 \\
\hline AIT3 & 82 & 74 & 51 \\
\hline
\end{tabular}

\section{Group $\times$ Stimulus Set Interaction}

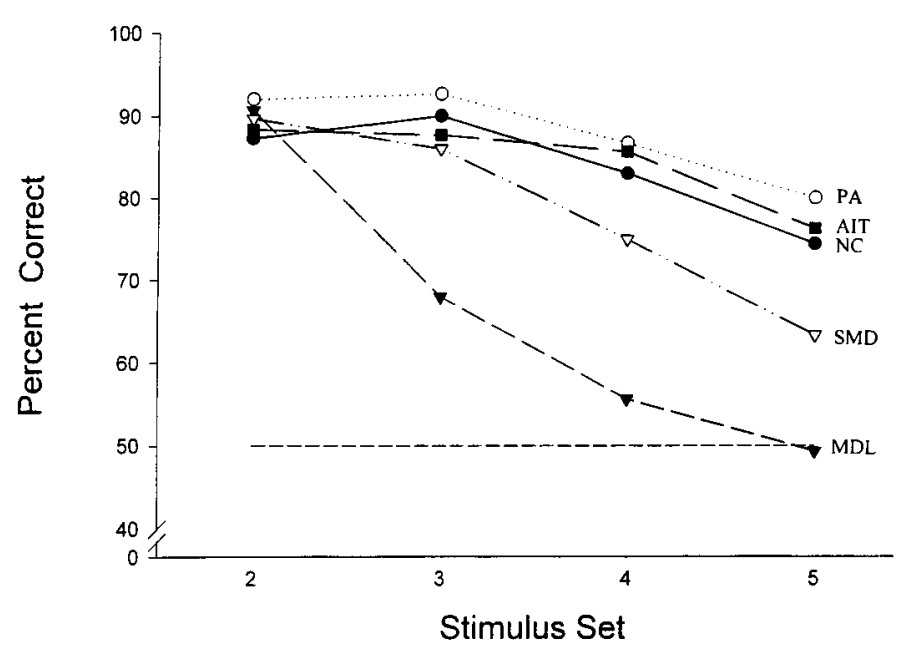

Figure 9. Graph showing mean performance of each group on the twothree-, four-, and five-stimulus sets. The level of chance performance $(50 \%)$ is indicated by the horizontal dotted line. Abbreviations as in Figure 8.

(SMD). In comparison with the NC group, the MDL group was impaired at the three-stimulus set (Newman-Keuls test, $p<0.001$ ) and was performing at chance at the four-stimulus $(p<0.001)$ and five-stimulus set $(p<0.001)$. The SMD group, on the other hand, was impaired only on the five-stimulus condition $(p<0.01)$, and performance was still above chance.

It is important to note that there was a significant drop in the performance of the normal animals (NC group) as the stimulus set in the presentation trials increased ( $p<0.025$ for set 2 vs set 5 and $p<0.001$ for set 3 vs set 5). Clearly, this decrease in normal performance would not have occurred had the animals attended only to the object chosen and ignored the other stimuli. The drop in normal performance when the set increased indicates that the
Table 2. Percentage correct performance on the stimulus set conditions

\begin{tabular}{lllll} 
Group & Two stimuli & Three stimuli & Four stimuli & Five stimuli \\
\hline NC group & & & & \\
NC1 & 87 & 87 & 84 & 72 \\
NC2 & 90 & 84 & 84 & 79 \\
NC3 & 88 & 93 & 79 & 71 \\
NC4 & 84 & 96 & 85 & 76 \\
PA group & & & & \\
PA1 & 97 & 95 & 87 & 87 \\
PA2 & 96 & 96 & 89 & 74 \\
PA3 & 83 & 87 & 84 & 79 \\
MDL group & & & & \\
MDL1 & 95 & 61 & 52 & 53 \\
MDL2 & 95 & 70 & 55 & 48 \\
MDL3 & 82 & 73 & 60 & 47 \\
SMD group & & & & \\
SMD1 & 87 & 89 & 73 & 64 \\
SMD2 & 89 & 87 & 76 & 65 \\
SMD3 & 93 & 82 & 76 & 61 \\
AIT group & & & & 79 \\
AIT1 & 93 & 88 & 84 & 76 \\
AIT2 & 82 & 83 & 88 & \\
AIT3 & 90 & 92 & & \\
\hline & & & & \\
\hline
\end{tabular}

earlier training on the self-ordered tasks was effective in making the animals attend not only to the object chosen on the presentation trials but also to the other objects in the set that were not chosen. Thus, the animals were indeed monitoring all stimuli in the set.

\section{DISCUSSION}

In the present experiment, the standard version of the self-ordered task was modified so that the delay during which the information must be maintained and the number of stimuli to be monitored could be manipulated independently. In all test trials, the monkey was faced with a choice between the object that it had selected on the preceding presentation trial and one of the objects that it had not selected. Thus, only the difference between the two manipulations (length of delay vs set size) can account for the observed differences in performance (Figs. 8,9). The results demonstrated a double dissociation between the effects of lesions restricted to the mid-dorsolateral prefrontal cortex and those of the anterior inferotemporal cortex in visual working memory. It has been shown in an unambiguous way that the critical variable determining the impairment in visual working memory after mid-dorsolateral prefrontal lesions is the number of stimuli that have to be monitored, whereas the critical variable after anterior inferotemporal lesions is the length of time during which the information must be maintained in memory. In other words, while the anterior inferotemporal region is a neuronal module necessary for the maintenance in memory of visual object information, the mid-dorsolateral prefrontal region is a module necessary for the application of an executive process that is here referred to as monitoring of information.

Working memory, like all cognitive processing, is the result of neuronal computations in multiple interacting brain structures. The challenge is to specify the contribution of each of these structures. The present dissociation is consistent with a theoretical model of the role of several neural structures in working memory that was proposed in the early 1990s [for details, see Petrides (1994)]. According to this model, basic short-term memory functions, such as the temporary storage and immediate processing of incoming and recalled information, are the result of computations performed within sensory-specific and multimodal parietal and temporal as- 
sociation areas that are the end points in systems devoted to the processing of sensory information. Transient neuronal firing in these posterior cortical association areas in the absence of the original stimuli represents the maintenance of the final stages of perceptual processing or the reactivation of previously stored representations, i.e., in both cases memories of the stimuli. Consistent with this view, lesions of the anterior inferotemporal cortical region, which is the end stage in the processing of visual object information, produced a failure to maintain such information as delays were increased; i.e., these lesions degraded visual short-term memory.

According to the above theoretical model, neuronal firing during the delay in the mid-dorsolateral prefrontal region, unlike in the anterior inferotemporal cortex, is not the maintenance of perceptual representations per se, but rather the maintenance of symbolic coding of the different cognitive representations (e.g., stimuli, events, thoughts, etc.) that must be acted on for the needs of the specific control operation (i.e., monitoring) that is performed there. The mid-dorsolateral prefrontal neuronal activity codes symbolically the different members of a current or an expected set of stimuli, and interactive modulations of activity between the pools of neurons coding the different stimuli act as markers of the current status of the different members of the set relative to each other or the occurrence/nonoccurrence of stimuli from the expected set. In this manner, multiple events and their relative status in current awareness can be monitored simultaneously. This kind of activity would be expected to occur at the study phase of a trial in a working memory task and would be maintained throughout the delay period so that any changes in the status of the stimuli can be marked by further modulations of that neuronal activity. Monitoring as defined here is necessary for the manipulation of cognitive representations (i.e., high-level planning) because the sine qua non for manipulation is the constant updating of the current relative status of intended acts or events in working memory.

The above view of the mid-dorsolateral prefrontal cortex received strong support from the present results. The increase in the requirement to consider multiple stimuli simultaneously (as the stimulus-set increased) proved devastating to the animals with mid-dorsolateral prefrontal lesions (Fig. 9). Note that when this monitoring requirement was minimal and delay was increased, the animals with mid-dorsolateral prefrontal lesions, unlike those with anterior inferotemporal lesions, were able to maintain the information in working memory as well as the control animals. The latter finding is consistent with earlier reports that monkeys with mid-dorsolateral prefrontal lesions (Petrides, 1991, 1995) or even larger dorsolateral prefrontal lesions (Bachevalier and Mishkin, 1986) performed as well as the control animals on delayed object recognition. Thus, after mid-dorsolateral prefrontal lesions, the problem did not lie in selectively attending to a stimulus and ignoring others or in processing that stimulus and then maintaining its representation during the delay, but rather in attending to the fact that stimulus A was selected and simultaneously noting that stimuli B, C, and D were not selected. In other words, the middorsolateral prefrontal lesions degraded neuronal activity that is necessary to code multiple events and their status relative to each other in current awareness.

The present results demonstrate that the maximum deficit in the monitoring of visual nonspatial information (Fig. 9) is obtained with rather complete lesions of the mid-dorsolateral prefrontal cortex (i.e., the MDL lesions) that include both its granular (areas 46D and 9/46D) and dysgranular (area 9) parts (Figs. 1B, 2). Lesions limited to the superior dysgranular part of the middorsolateral prefrontal cortex (i.e., the SMD lesions) demonstrated a significant impairment in the five-stimulus condition and performed as well as the control group with three stimuli (Fig. 9). These latter findings are consistent with two recent studies (Petrides, 1998; Levy and Goldman-Rakic, 1999) in which monkeys with lesions restricted mostly to the superior part of the middorsolateral prefrontal cortex (i.e., area 9) and sparing the granular cortex just above the sulcus principalis were shown not to be severely impaired on the nonspatial self-ordered task with three objects.

Interestingly, in a recent study by Levy and Goldman-Rakic (1999), monkeys with lesions limited to the cortex lining the sulcus principalis (i.e., sulcal area 46), sparing the mid-dorsolateral prefrontal region studied here, performed well on the self-ordered task with three objects, although those monkeys were severely impaired on spatial delayed response tasks as would be expected from the large literature showing spatial impairments from lesions limited to the sulcus principalis (for review, see Petrides, 1994). Thus, the effects of lesions limited to the cortex within the sulcus principalis (i.e., sulcal area 46) can be dissociated from those of the cortex that lies above the sulcus principalis (dorsal areas 46 and 9). However, the monkeys with sulcus principalis lesions in the Levy and Goldman-Rakic (1999) study were not tested with sets longer than three objects on the self-ordered test, and thus it remains an open question whether animals with such lesions would be impaired on longer sets.

The cortex in the caudal part of the sulcus principalis is part of area 8 and receives primarily visuospatial inputs from the parietal cortex (Barbas and Mesulam, 1981, 1985; Huerta et al., 1987; Andersen et al., 1990; Schall et al., 1995; Petrides and Pandya, 1999), and the ventral bank of the sulcus principalis receives primarily somatosensory inputs from the rostral parietal and the opercular parietal region (Petrides and Pandya, 1984; Barbas and Mesulam, 1985; Preuss and Goldman-Rakic, 1989). It has been argued previously (Petrides, 1991, 1994; Petrides and Pandya, 1999) that lesions limited to this part of the sulcus principalis, which receives primarily visual and somatosensory spatial input, might result in a purely spatial impairment (e.g., spatial delayed response tasks) and disrupt the flow of critical spatial input into the multimodal monitoring system that lies in the mid-dorsolateral prefrontal region above the sulcus principalis. Because visual nonspatial input to the mid-dorsolateral prefrontal monitoring system would be provided from the ventrolateral prefrontal cortex (Petrides and Pandya, 1999), lesions limited to the sulcus principalis need not affect nonspatial self-ordered tasks. Thus, if the lesions are made at the points of entry of modality-specific information into the frontal cortex, modality-specific impairments could result.

The above findings are consistent with the results of functional neuroimaging studies in normal human subjects. Such studies have consistently shown increases within the mid-dorsolateral prefrontal region of the human brain during the performance of working memory tasks whenever monitoring is taxed, regardless of the modality of the information (for review, see Petrides, 2000). Interestingly, in a recent study that reported a signal specifically related to the maintenance of a spatial representation, this signal was located not in the mid-dorsolateral prefrontal cortex but in the caudal position of the superior frontal sulcus (Courtney et al., 1998) that most probably corresponds to area 8 or rostral area 6 (Petrides and Pandya, 1999). Thus, activity in the mid-dorsolateral prefrontal region is related to monitoring within working memory, regardless of whether the information is spatial or not (Petrides, 2000). Spatial delay-dependent activity can be selectively obtained in regions caudal to the mid-dorsolateral prefrontal region (Courtney et al., 1998).

\section{REFERENCES}

Andersen RA, Asanuma C, Essick G, Siegel RM (1990) Corticocortical connections of anatomically and physiologically defined subdivisions within the inferior parietal lobule. J Comp Neurol 296:65-113.

Bachevalier J, Mishkin M (1986) Visual recognition impairment follows ventromedial but not dorsolateral prefrontal lesions in monkeys. Behav Brain Res 20:249-261.

Barbas H, Mesulam M-M (1981) Organization afferent input to subdivisions of area 8 in the rhesus monkey. J Comp Neurol 200:407-431.

Barbas H, Mesulam M-M (1985) Cortical afferent input to the principalis region of the rhesus monkey. Neuroscience 15:619-637.

Courtney SM, Petit L, Maisog JM, Ungerleider LG, Haxby JV (1998) An area specialized for spatial working memory in human frontal cortex. Science 279:1347-1351.

D’Esposito M, Aguirre GK, Zarahn E, Ballard D, Shin RK, Lease J (1998) 
Functional MRI studies of spatial and nonspatial working memory. Cognit Brain Res 7:1-13.

Huerta MF, Krubitzer LA, Kaas JH (1987) Frontal eye fields as defined by intracortical microstimulation in squirrel monkeys, owl monkeys, and macaque monkeys. II. Cortical connections. J Comp Neurol 271:473-492.

Levy R, Goldman-Rakic PS (1999) Association of storage and processing functions in the dorsolateral prefrontal cortex of the nonhuman primate. J Neurosci 19:5149-5158.

Mishkin M (1982) A memory system in the monkey. Philos Trans R Soc Lond B Biol Sci 298:85-95.

Owen AM (1997) The functional organization of working memory processes within the human lateral frontal cortex: the contribution of functional neuroimaging. Eur J Neurosci 9:1329-1339.

Pandya DN, Kuypers HGJM (1969) Cortico-cortical connections in the rhesus monkey. Brain Res 13:13-36.

Pandya DN, Yeterian EH (1985) Architecture and connections of cortical association areas. In: Cerebral cortex (Peters A, Jones EG, eds), pp 3-61. New York: Plenum.

Pandya DN, Dye P, Butters N (1971) Efferent cortico-cortical projections of the prefrontal cortex in the rhesus monkey. Brain Res 31:35-46.

Petrides M (1987) Conditional learning and the primate frontal cortex. In: The frontal lobes revisited (Perecman E, ed), pp 91-108. New York: IRBN

Petrides M (1991) Monitoring of selections of visual stimuli and the primate frontal cortex. Proc R Soc Lond B Biol Sci 246:293-298.

Petrides M (1994) Frontal lobes and working memory: evidence from investigations of the effects of cortical excisions in nonhuman primates. In: Handbook of neuropsychology, Vol 9 (Boller F, Grafman J, eds), pp 59-82. Amsterdam: Elsevier.
Petrides M (1995) Impairments on nonspatial self-ordered and externally ordered working memory tasks after lesions of the mid-dorsal part of the lateral frontal cortex in the monkey. J Neurosci 15:359-375.

Petrides M (1998) Working memory and the mid-dorsolateral prefrontal cortex. Soc Neurosci Abstr 24:18.

Petrides M (2000) Mapping prefrontal cortical systems for the control of cognition. In: Brain mapping: the systems (Toga AW, Mazziotta JC, eds), pp 159-176. San Diego: Academic.

Petrides M, Pandya DN (1984) Projections to the frontal cortex from the posterior parietal region in the rhesus monkey. J Comp Neurol 228:105-116.

Petrides M, Pandya DN (1994) Comparative architectonic analysis of the human and the macaque frontal cortex. In: Handbook of neuropsychology, Vol 9 (Boller F, Grafman J, eds), pp 17-58. Amsterdam: Elsevier.

Petrides M, Pandya DN (1999) Dorsolateral prefrontal cortex: comparative cytoarchitectonic analysis in the human and the macaque brain and corticocortical connection patterns. Eur J Neurosci 11:1011-1036.

Preuss TM, Goldman-Rakic PS (1989) Connections of the ventral granular frontal cortex of macaques with perisylvian premotor and somatosensory areas: anatomical evidence for somatic representation in primate frontal association cortex. J Comp Neurol 282:293-316.

Schall JD, Morel A, King DJ, Bullier J (1995) Topography of visual cortex connections with frontal eye field in macaque: convergence and segregation of processing streams. J Neurosci 15:4464-4487.

Walker AE (1940) A cytoarchitectural study of the prefrontal area of the macaque monkey. J Comp Neurol 73:59-86. 